\title{
MD Study of Energetics, Melting and Isomerization of Aluminum Microclusters
}

\author{
Mustafa Böyükata ${ }^{a}$ and Ziya B. Güvenç ${ }^{b}$ \\ ${ }^{a}$ Department of Physics, Erciyes University, 66100 Yozgat, Turkey \\ ${ }^{b}$ Department of Electronic and Communication Engineering, Çankaya University, 06530 Ankara, Turkey
}

Received on 7 September, 2005

\begin{abstract}
Voter and Chen version of an Embedded Atom Model has been applied to study the locally stable structures, energies, melting, isomerization and growth patterns of small aluminum clusters, $A l_{n}$, in the size range of $n=2-13$. Using molecular dynamics and thermal quenching simulations, the global minima and the other locally stable structures have been distinguished from those stationary structures that correspond to saddle points of the potential energy surface. A large number (10000) of independent initial configurations generated at high temperatures has been used to obtain the stable isomers, and the probabilities of sampling different basins of attractions, for each size of the clusters. Their energy spectra have been determined and melting, and isomerization dynamics are investigated.
\end{abstract}

Keywords: Aluminum; Cluster; Molecular dynamics

\section{INTRODUCTION}

The study of metallic clusters received an increasing attention in the recent years because of many technological applications [1-5]. The nature of their chemical/physical properties provides a rich source for experimental and theoretical studies. Investigations on structures and melting properties of atomic clusters are important due to their roles as building blocks for new nanomaterials, between molecules and bulk [612]. A systematic study of evolution of these properties with size allows elucidation of the transition from the molecular structure to condensed matter phase. Furthermore, clusters play an important role in many chemical reactions as catalysts since the structure of small metal clusters in a reaction can have a major effect on the rate of formation of products $[13,14]$ because of the larger surface to volume ratio. Phases and phase changes of finite systems have been studied extensively, as well $[15,16]$.

In this study the $A l$ clusters are characterized by using a semi-empirical Voter and Chen [17] version of the embedded atom method (EAM). The EAM, which requires 2 to 5 times the work of a pair potential includes many-body effects that are important for description of metallic cohesion, is more adequate for the metallic systems. The virtue of Voter and Chen version is that it is derived by fitting the EAM to both the basic bulk properties of the metal and to the bond-length and bondenergy of the diatomic molecule, $A l_{2}$, simultaneously [18-20]. So, this EAM may have a wider range of validity from a few atoms to the larger sizes of the $A l_{n}$ clusters. The EAM grasps the major parts of the interatomic interactions and gives accurate structural and energetic information on various extended and truncated systems [21-23]. In the literature Garcia-Rodeja et al. [23] used the Voter and Chen version of the EAM by carrying out molecular dynamics (MD) simulations to study the structures and melting of $6 \mathrm{fcc}$ transition metals including $A l$ clusters $(N i, P d, A l, C u, A g$, and $A u)$ in the size range of the $n=2-23$. However, they did not present any information about the stable isomeric forms and their sampling probabilities.

We have carried out MD and thermal quenching (TQ) sim- ulations to study not only the lowest energy structures of the $A l_{n}$ clusters, but also their number of stable isomers for the size range of $n=2-13$. In addition, the clusters' growth pathway, probabilities of sampling the basins of attraction of the stable isomers, and their energy spectrum-widths (ESW) which are defined by the energy difference between the most and the least stable isomers, have been analyzed. The melting process (phase changes from solid to liquid forms) has also been investigated, and the underlying mechanisms are discussed. In addition temperature dependence of the number of isomers for the $n=7,10$, and 13 clusters are investigated. The computational method, described in Section II, combines the interaction potential and the computational procedure. The results and discussions are presented in Section III, and a brief summary of our conclusions is given in Section IV.

\section{THEORETICAL BACKGROUNDS}

In any $N$-scaling energy expression, the total energy of an $\mathrm{N}$-atom cluster in the EAM is given by a sum of the total interaction energies of all the atoms

$$
V_{E A}=\sum_{i}\left[F_{i}\left(\bar{\rho}_{i}\right)+\frac{1}{2} \sum_{j \neq i} \phi_{i j}\left(r_{i j}\right)\right]
$$

where $\bar{\rho}_{i}$ is the effective host electron density at the atom- $i$ site, and it is obtained by a linear superposition of the spherically symmetric atomic density $\rho_{i}$,

$$
\begin{gathered}
\bar{\rho}_{i}=\sum_{i} \rho_{j}\left(r_{i j}\right), \\
\rho_{j}(r)=r^{6}\left(e^{-\beta r}+2^{9} e^{-2 \beta r}\right) .
\end{gathered}
$$

The $F_{i}\left(\bar{\rho}_{i}\right)$ is the embedding energy function - energy required to embed atom- $i$ into the background electron density at site $i$ which provides a many body interaction contribution to the energy. The $\phi_{i j}\left(r_{i j}\right)$ is a core-core, formed by the remaining charges, interaction between atoms $i$ and $j$ separated by a distance of $r_{i j}$. The sums over neighboring atoms $j$ are limited by the range of the cutoff $(\sim 4.7 \AA)$ for $\phi_{i j}\left(r_{i j}\right)$ and $\rho_{i}$. The EAM 
model of Voter and Chen has a medium-range attractive contribution in the core-core interaction. All the parameters in the model were determined by minimizing the root-mean-square deviation between the calculated and experimental values of the elastic constants, and the unrelaxed vacancy formation energy of the bulk material and of the bond-length and bondstrength of the diatomic molecule. The experimental data used in the fitting process along with the calculated values can be found in Ref. [17].

In this study, the equilibrium structural forms of the $A l_{n}$, $n=2-13$ clusters have been obtained using the MD and TQ simulations. Hamilton's equation of motion were solved for all the atoms in a cluster using Hamming's modified $4^{\text {th }}$ order predictor-corrector propagator with a step-size of $4 \times 10^{-16}$ $\mathrm{s}$, which guarantees conservation of total energy of the clusters to within $0.01 \%$. The dynamical properties of the clusters at different fixed values of their total energies were extracted from the simulation runs of $4 \times 10^{6}$ steps. The melting behaviour of the clusters was investigated by the root-mean-square (RMS) bond-length fluctuation, $\delta$,

$$
\delta=\frac{2}{N(N-1)} \sum_{i<j} \frac{\left(<r_{i j}^{2}>-<r_{i j}>^{2}\right)^{1 / 2}}{<r_{i j}>} .
$$

Clusters are prepared at temperature, $T(K)$, as

$$
T(K)=\frac{2<E_{k}>}{(3 n-6) k}
$$

where $k$ is the Boltzmann constant, $E_{k}$ is the total kinetic energy of the cluster, the $\langle>$ denotes the long-time average, and the $n$ is the number of atoms. In order to obtain isomer statistics, 10000 independent initial configurations are generated by recording of phase-space coordinates of the clusters at every $500 \mathrm{MD}$ simulation steps along high-energy trajectories. The internal energies were chosen to be high enough (over $2000 \mathrm{~K}$ ) to "melt" the clusters, so that, one can sample as many different points as possible in the $6 \mathrm{~N}$ dimensional phase-space. The TQ of these configurations were completed by quenching at every 30 simulation steps. This was achieved by setting the internal kinetic energies of the clusters to zero at every 30 simulation steps until the internal kinetic energies were completely removed - this process of quenching eliminates all the meta-stable forms of the isomers.

\section{RESULTS AND DISCUSSION}

For the $A l_{n}, n=2-13$, clusters studied we have performed a series of MD simulations. The random search method has been used to find the global minima for the $A l$ clusters with up to 13 atoms, bound by the EAM potential. After quenching 10000 different phase-space configurations which were prepared at high temperature (over $2000 \mathrm{~K}$ ) near fragmentation points, the global minimum energy geometries of the $A l_{2}-A l_{13}$ clusters are obtained and presented in Fig. 1. The bond-energy and the bong-length of the $A l_{2}$ dimer are $-0.77 \mathrm{eV} /$ atom and $2.44 \AA$, respectively (these experimental values were used in the fitting procedure of the EAM).

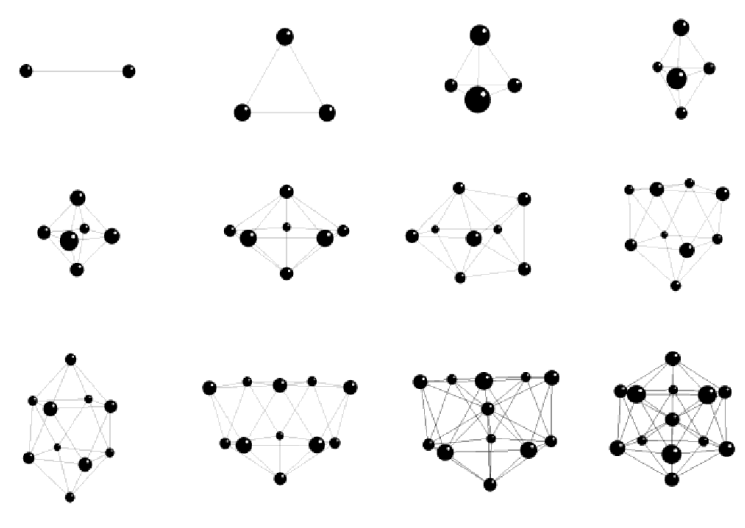

FIG. 1: Stable structures of the $A l_{2}-A l_{13}$ clusters.

The lowest energy structure of the $A l_{3}$ cluster is an equilateral triangle with a bond length of $2.52 \AA$, and energy of -1.5 $\mathrm{eV} /$ atom. Tetrahedral is found to be the lowest energy structure for an $A l_{4}$, with a bond length of $2.58 \AA$, and a binding energy of $-1.59 \mathrm{eV} /$ atom. The least energy structure of the $A l_{5}$ belongs to a trigonal bipyramid group. The bond length is $2.58 \AA$, and the binding energy of the $A l_{5}$ cluster is calculated as $-1.79 \mathrm{eV} /$ atom. The largest bond distance is obtained for the $A l_{6}$, and it has a bipyramid form with a bond length of 2.61 $\AA$, and a binding energy of $-1.98 \mathrm{eV} /$ atom. Each of the clusters, $n>6$, has one or more of the trigonal, tetragonal, pentagonal bipyramid forms as a main sustaining part. Therefore, it is possible to classify them with respect to their backbones. We call $A l_{2}-A l_{4}$ group as primitive. The tetragonal bipyramid symmetry is observed in the lowest energy structures of the $A l_{6}$ and $A l_{8}$ clusters. The $A l_{7}$ cluster belongs to pentagonal bipyramid group. The pentagonal bipyramid form is the most stable structure of the $A l_{7}$ clusters. This structure forms the backbone of the icosahedral symmetry with the binding energy of $-2.078 \mathrm{eV} /$ atom. The lowest energy structure of the $A l_{8}$ can be constructed from the minimum energy structure of the $A l_{6}$ by capping the symmetric two faces of the bipyramid with respect to the equatorial plane, and its energy is -2.162 $\mathrm{eV} /$ atom. The minimum energy structures of the $A l_{9}$ and $A l_{10}$ clusters are constructed from the tetragonal pyramid forms. It is possible to construct the $A l_{10}$ from the previous size (without changing much its existing structure) by simply capping the upper surface with an atom. They have two 4-atom rings in their geometries. The lowest energy form of the $A l_{11}$ has two pentagonal rings. The $A l_{12}-A l_{13}$ clusters have the pentagonal bipyramid as their backbones. As seen, it is possible to construct the structure of the $A l_{13}$ from that of the $A l_{12}$ by simply adding an atom without changing the existing symmetry. The lowest energy structure of the $n=13$ has a particular importance since it is the first of so called magic numbers, and it is an icosahedron - a central atom is surrounded by 12 atoms, which form a spherical shell with a calculated radius of $2.60 \AA$.

The number of isomers, the lowest binding energies per atom, and the ESW for the $n=7-13$ are given in Table I. As expected, the number of isomers increases as the cluster 
TABLE I: Number of isomers and energetics of the $A l_{7}-A l_{13}$ clusters obtained from 10000 phase space coordinates.

\begin{tabular}{rccc}
\hline \hline$N$ & Number of isomers & $\begin{array}{c}E(\mathrm{eV} / \text { atom }) \text { of } \\
\text { the stable structure }\end{array}$ & $\begin{array}{c}\text { ESW } \\
(\mathrm{eV} / \text { atom })\end{array}$ \\
\hline 7 & 4 & -2.078 & 0.077 \\
8 & 4 & -2.162 & 0.064 \\
9 & 10 & -2.232 & 0.106 \\
10 & 20 & -2.287 & 0.111 \\
11 & 39 & -2.318 & 0.109 \\
12 & 65 & -2.377 & 0.124 \\
13 & 135 & -2.467 & 0.176 \\
\hline \hline
\end{tabular}

size increases due to the high dimensionality of the configuration space. The ESW per atom increases from $n=8$ to 10 and $n=11$ to 13 . In these two different intervals the clusters have two different 'mother' geometries, $A l_{6}$ and $A l_{7}$, respectively.

Results for the dynamical properties and phase changes of the 3 selected clusters $\left(A l_{7}, A l_{10}\right.$ and $\left.A l_{13}\right)$ are presented in Fig. 2.

The starting structures of the clusters $(n=7,10$, and 13) are in their global minimum energy forms. In order to see the phase change properties of the stable structures, the RMS bond-length fluctuation curves are plotted as a function of the temperature for these three structures in Fig. 2 (top). These curves are formed by calculating d values over $4 \times 10^{6}$ simulation steps at different total energies (total energies are increased in a stepwise manner). The melting points of these three clusters are lower than that of the bulk system (melting points are extracted from the middle points of the jumps observed in the RMS curves). It is also possible to determine the melting temperature using the stability functions $(S)$ of the RMS fluctuations,

$$
S=\frac{\delta_{m-1}-\delta_{m+1}}{2}-\delta_{m}
$$

Here, the $m$ refers to each of the RMS values. The changes in the stability functions are monitored for the $n=7,10$ and 13, and presented in Fig. 2 (middle). The fluctuations in $S$ illustrate phase transformation from the solid-like to liquidlike forms, and the transition temperature intervals for each of the clusters (width of the fluctuations). The first peak shows the end of solid like, and the last one defines the full liquid like behaviors of the clusters. In addition, isomerizations start from the beginning of the melting process (beginning of the fluctuations in $S$ ). As seen, the melting temperature increases as the cluster's size increases. However, they are all smaller than the bulk melting temperature.

We also give the changes in the number of isomers as a function of the temperature of these three structures in Fig. 2 (bottom). In order to see the increase in the number of isomers as a function of the internal kinetic energy of the clusters, 5000 phase-space coordinates were prepared at all temperature steps from 0 to over $2000 \mathrm{~K}$. Using the TQ technique numbers of the isomers have been determined. The $A_{7}$ (and also for the $A l_{8}$ ) cluster has four stable isomers. All these isomers can be obtained within the phase transformation interval from solid to liquid form. However, in this interval, just three of the isomers of the $A l_{8}$ are observed, and the fourth one is determined above $1400 \mathrm{~K}$. On the other hand, the number of isomers increases sharply for the $n \geq 9$. For the $A l_{9}$ there is a threshold value of the number of stable isomers at near 1600 $K$. As seen, the number of isomers also reaches to a threshold value for the $n=13$ above $2000 K$. At the lower temperatures, the numbers are equal to 1 since the clusters are in the potential energy wells of their global minima. As a result, it is important to run trajectories longer for any size of the clusters, and record as many numbers of the phase- space coordinates as possible at very high temperature (near the fragmentation point).

In order to test the result obtained with 5000 different configurations, 10000 independent phase-space coordinates are sampled by using the TQ simulations for all the sizes considered at high temperature, over $2000 \mathrm{~K}$. As seen, even though 5000 samples capture many isomers, it is better to use much more phase-space configurations for the sampling. Especially, this is more important for the larger clusters $(n>13)$ because of their higher dimensionalities which lead to produce more stable isomers. In addition, quenching from the higher temperature also yields larger number of stable isomers. The total numbers of the stable isomers are presented in Table I. From these TQ processes for all the sizes the isomer forming probabilities are obtained. Although the total energy of the most stable structure of the $A l_{7}$ is less than that of the second one, the sampling probability of the $2^{\text {nd }}$ isomer is higher than the others (not shown). This means that, probability of finding the final configuration of the hot molten cluster reached at the end of the cooling process, the energy value of the quenched stable isomer is not the only control parameter. The other parameters may affect the probabilities are the number of accessible states (identical configurations) of the isomer at its lowest energy configuration, the backbones, and the symmetry of the clusters. However, for many cluster sizes, the most stable structure is the most probable one. Generally, as the total energy of the locally stable structures increases for a given size $(n>8)$, the backbones of the structures change from one of the hexagonal, pentagonal, or tetragonal forms to mostly trigonal bipyramid form.

There are some energy bands, in which, a number of energetically close isomers are present with smoothly decreasing probabilities, and there are some energy gaps, in which, no isomers are observed. The backbones are responsible for the energy bands and the energy gaps in the isomer energy spectrum probability distribution (see Fig. 3).

The effect of the symmetry is the best seen for the $n=13$. The probability distribution graph of the $A l_{13}$ differs from the others (not shown). In this size, the probability of the lowest energy structure is much higher than those of the other isomers because of the perfect spherical symmetry of the icosahedron. The isomer forming probability distribution is fitted by an exponential function of the form;

$$
P(E)=a e^{\left\{\frac{b}{E+\left|E_{h}\right|}\right\}}
$$

where, $a>0$ : the dimensionless constant (approximately probability of formation of the lowest energy structure), $b>0$ : 


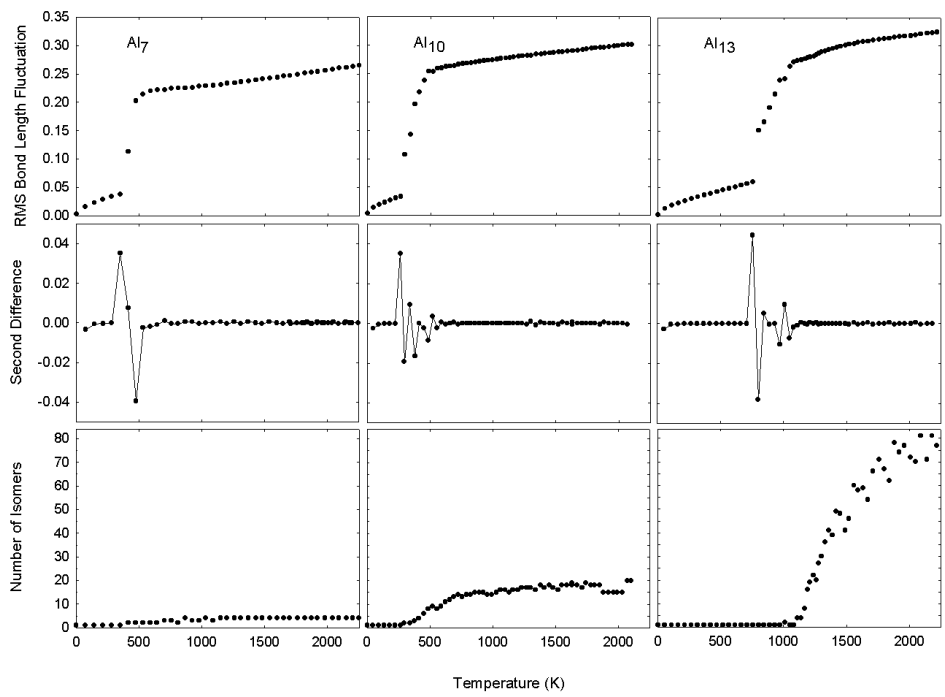

FIG. 2: Temperature dependent changes in the RMS-bond length fluctuations, stability functions for RMS-fluctuations, and isomers of $A l_{7}, A l_{10}, A l_{13}$ clusters.

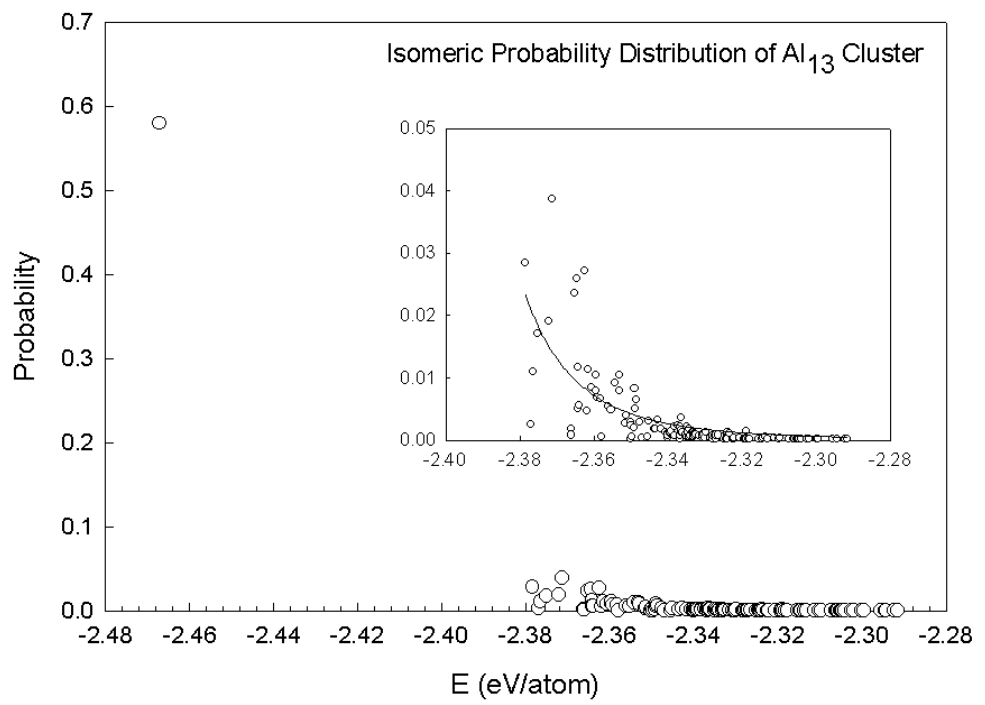

FIG. 3: Isomeric probability distribution for the $A l_{13}$ cluster. Inset shows all the points except the probability of the lowest energy structure and behavior of the exponential function, Eq. (6).

scaling factor in units of $e V, E_{h}$ is the energy of the energetically highest isomer, and $E<0$ is the internal energy of a quenched isomer $[24,25]$ (starts from the first isomer). The exponent, as seen, is inversely proportional with the energy of the isomer. For all the sizes the probability distributions as a function of the energy nearly obeys the equation with exclusion of some of the points in the distributions.

\section{CONCLUSIONS}

A computational analysis has performed for investigating structures, energetics, melting and isomerization dynamics of small $A l$ clusters. The MD and TQ simulations have been carried out to search the global and the other locally stable structures, the number of isomers, isomer statistics, and energy spectrum widths. All locally stable structures of the $A l_{n}$ clusters $(n=5-13)$ have either one or more of the trigonal, tetragonal, pentagonal, or hexagonal bipyramids as their backbones. It is found that, in general, isomers sampling probability distributions fall exponentially as the total energies of the isomers increase. However, the energy dependence of the probabilities is not simple. The lowest energy structure is not always the most probable isomer. The minima found in this study can be good starting points for comparing the results obtained with different potentials. The 
results we report here, at least, represent this opportunity for the $A l$ clusters described by Voter and Chen [17] version of the EAM in the size range of $n=2-13$. Finally, the present study shows that investigating the numbers and the structures of the stable isomers can provide a deeper understanding of the clusters' growth paths, the energy spectrum widths, energy bands and gaps formed by the isomers. Moreover, it is observed that in order to find all possible stable structures for any cluster size, the preparation of the phase-space coordinates must be done at very high temperature (near the fragmentation point), and it must consist of a sufficient number of samples for the TQ process.

\section{Acknowledgements}

This work was supported by Erciyes University Research Fund (Project Number: FBA.03.29).
[1] B. K. Rao, S. N. Khanna, and P. Jena, J. Cluster Sci. 10, 477 (1999).

[2] R. O. Jones, Phys. Rev. Lett. 67, 224 (1991).

[3] B. K. Rao and P. Jena, J. Chem. Phys. 111, 1890 (1999).

[4] M. D. Deshpande, D. G. Kanhere, I. Vasiliev, and R. M. Martin, Phys. Rev. B 68, 035428 (2003).

[5] P. Mitev, D. G. Papageorgiou, Ch. E. Lekka, and G. A. Evangelakis, Surf. Sci. 566-568, 937 (2004).

[6] H. Haberland (Ed.), Clusters of atoms and molecules (Springer, Berlin, 1994).

[7] G. Schmid (Ed.), Clusters and colloids, (VCH, Weinheim, 1994).

[8] T. P. Martin (Ed.),Large clusters of atoms and molecules (Kluwer, Dordrecht, 1996).

[9] J. Jellinek (Ed.),Theory of atomic and molecular clusters (Springer, Berlin, 1999).

[10] H. Cox, R. L. Johnston, and J. N. Murrell, J. Sol. Stat. Chem. 145, 517 (1999).

[11] R. Alrichs and S. D. Elliott, Phys. Chem. Chem. Phys. 1, 13 (1999).

[12] L. D. Lloyd and L. R. Johnston, J. Chem. Soc., Dalton Trans., 307 (2000).

[13] J. Jellinek and Z. B. Güvenç, Z. Phys. D 26, 110 (1993).

[14] J. Jellinek and Z. B. Güvenç, in The Synergy Between Dynam- ics and Reactivity at Clusters and Surfaces, ed. L. J. Farrugia (Kluwer, Dordrecht, 1995), p.217.

[15] Y. J. Lee, R. M. Nieminena, E.-K. Lee, and S. Kim, Comp. Phys. Comm. 142, 201 (2001).

[16] S. Darby, T. V. M. Janes, R. L. Johnston, and C. Roberts, J. Chem. Phys. 116, 1536 (2002).

[17] A. F. Voter, Los Alamos Unclassified Technical Report \# LAUR 93-3901 (1993)

[18] Z. B. Güvenç, and J. Jellinek, Z. Phys. D 26, 304 (1993).

[19] Z. B. Güvenç, J. Jellinek, and A. F. Voter, Physics and Chemistry of Finite Systems: From Clusters to Crystals, Vol.1 (Kluwer Academic Publishers, Dordecht (1992), p.411.

[20] M. Böyükata, Z. B. Güvenç, S. Özçelik, P. Durmuş, and J. Jellinek, Int. J. Quantum Chem. 84, 208 (2001).

[21] Y. Mishin, D. Farkas, M. J. Mehl, and D. A. Papaconstantopoulos, Phys. Rev. B 59, 3393 (1999).

[22] V. G. Grigoryan and M. Springborg, Chem. Phys. Lett. 375, 219 (2003).

[23] J. Garcia-Rodeja, C. Rey, and L. J. Galleo, Phys. Rev. B 49, 8495 (1994).

[24] Z. B. Güvenç and D. Güvenç, J. Jellinek, Math. Comp. Appl. 4, 75 (1999).

[25] A. Sebetçi and Z. B. Güvenç, Surf. Sci. 525, 66 (2003). 